\title{
LIOUVILLE THEOREMS FOR STATIONARY FLOWS OF SHEAR THICKENING FLUIDS IN 2D
}

\author{
Guo Zhang \\ Central China Normal University, School of Mathematics and Statistics \\ Wuhan, P. R. China; guozhang625@hotmail.com
}

\begin{abstract}
In this paper we consider entire weak solutions $u$ of the equations for stationary flows of shear thickening fluids in the plane and prove Liouville theorems in case of finite energy or under suitable integrability assumptions on $u$.
\end{abstract}

\section{Introduction}

In this paper, we prove different types of Liouville theorems for entire weak solutions $u: \mathbf{R}^{2} \rightarrow \mathbf{R}^{2}, \pi: \mathbf{R}^{2} \rightarrow \mathbf{R}$ of the following system

$$
\left\{\begin{array}{l}
-\operatorname{div}[T(\varepsilon(u))]+u^{k} \partial_{k} u+D \pi=0 \\
\operatorname{div} u=0 \text { in } \mathbf{R}^{2}
\end{array}\right.
$$

which describes the stationary flow of an incompressible generalized Newtonian fluid. In equation (1.1), $u$ denotes the velocity field, $\pi$ the pressure function, $u^{k} \partial_{k} u$ the convective term, and $T$ represents the deviatoric part of the stress tensor. As usual $\varepsilon(u)$ is the symmetric derivative of $u$, i.e. $\varepsilon(u)=\frac{1}{2}\left(D u+(D u)^{T}\right)=\frac{1}{2}\left(\partial_{i} u^{k}+\partial_{k} u^{i}\right)_{1 \leq i, k \leq 2}$.

Assume that the tensor $T$ is the gradient of a potential $H: S^{2} \rightarrow \mathbf{R}$ defined on the space $S^{2}$ of all symmetric $(2 \times 2)$ matrices, where $H$ is of the special form

$$
H(\varepsilon)=h(|\varepsilon|) \text {, }
$$

for a nonnegative function $h$ of class $C^{2}$. Thus we have

$$
T(\varepsilon)=D H(|\varepsilon|)=\mu(|\varepsilon|) \varepsilon, \quad \mu(t)=\frac{h^{\prime}(t)}{t},
$$

which means that the viscosity coefficient may depend on $\varepsilon$, and system (1.1) describes the motion of a generalized Newtonian fluid. For further mathematical and physical explanations, we refer to Ladyzhenskaya [Lad69], Galdi [Gal94a, Gal94b], Malek, Necas, Rokyta and Ruzicka [MNRR96], and Fuchs and Seregin [FS00]

If $\mu(t)$ is an increasing function, we call the fluid shear thickening. If $\mu(t)$ is a decreasing function, we call the fluid shear thinning. Clearly, if $\mu(t)=$ constant, then (1.1) reduces to the stationary Navier-Stokes equations for incompressible Newtonian fluids.

The study of Liouville type theorems for Navier-Stokes equations goes back to the work of Gilbarg and Weinberger [GW78]. They showed that entire solutions $u$ of the stationary Navier-Stokes equations in the plane are constants under the

doi:10.5186/aasfm.2015.4052

2010 Mathematics Subject Classification: Primary 76D05, 76D07, 76M30, 35Q30.

Key words: Stationary flows, shear thickening fluids, entire weak solutions, Liouville theorems. 
condition $\int_{\mathbf{R}^{2}}|D u|^{2} d x<\infty$. For the unstationary backwards Navier-Stokes equations in 2D, recently, Koch, Nadirashvili, Seregin and Sverak [KNSS09] showed that $u(x, t)=b(t)$ on $\mathbf{R}^{2} \times(-\infty, 0)$ provided the solutions are bounded. Clearly, this result implies Liouville's theorem for the stationary Navier-Stokes equations, that is, bounded solutions to the stationary Navier-Stokes equations are constants.

For degenerate power law fluids, Bildhauer, Fuchs and Zhang [BFZ13] got similar Liouville type theorems as described above. For the stationary Navier-Stokes equation, they showed that $u(x)$ is a constant provided $u(x)=o\left(|x|^{\alpha}\right)$, for some $\alpha<\frac{1}{3}$ at infinity. Later, Jin and Kang [JK14] improved the regularity of weak solutions and used their result to get partially similar Liouville type theorems. For a survey of Liouville type theorems for generalized Newtonian fluids, the readers are recommended to see the paper [Fuc14].

In the whole paper, we will concentrate on the following type of shear thickening fluids with potential $h$ satisfying the next conditions:

$$
\begin{aligned}
& h \text { is strictly increasing and convex } \\
& \text { together with } h^{\prime \prime}(0)>0 \text { and } \lim _{t \rightarrow 0} \frac{h(t)}{t}=0 .
\end{aligned}
$$

(doubling property) there exists a constant $a>0$

such that $h(2 t) \leq a h(t)$ for all $t \geq 0$.

$$
\text { we have } \frac{h^{\prime}(t)}{t} \leq h^{\prime \prime}(t) \text { for any } t \geq 0 \text {. }
$$

For general potentials $h$ satisfying (A1)-(A3), very recently Fuchs [Fuc12b] showed that bounded solutions $u$ of (1.1) must be constants provided $u$ satisfies the asymptotic condition $\left|u-u_{\infty}\right| \rightarrow 0$ at infinity, for some vector $u_{\infty} \in \mathbf{R}^{2}$. Later, the author [Zha13] removed the above assumption on $u$ at infinity and showed that any bounded solution $u$ of (1.1) must be a constant vector .

Under the hypothesis that the flow is slow (which means that the convective term vanishes ) and that energy is finite, i.e. $\int_{\mathbf{R}^{2}} h(|D u|) d x<\infty$, Fuchs [Fuc12b] showed that the velocity field $u$ is a constant vector. In this note, we remove the additional constraint that the flow is slow and prove the following theorem which is an extension of the result of Gilbarg and Weinberger in the setting of shear thickening fluids.

Theorem 1.1. Let $u \in C^{1}\left(\mathbf{R}^{2}, \mathbf{R}^{2}\right)$ be an entire weak solution to (1.1) i.e.

$$
\int_{\mathbf{R}^{2}} T(\varepsilon(u)): \varepsilon(\varphi) d x-\int_{\mathbf{R}^{2}} u^{k} u^{i} \partial_{k} \varphi^{i} d x=0
$$

for all $\varphi \in C_{0}^{\infty}\left(\mathbf{R}^{2}, \mathbf{R}^{2}\right)$ with $\operatorname{div} \varphi=0$, and let $u$ satisfy the condition $\int_{\mathbf{R}^{2}} h(|D u|) d x$ $<\infty$. Then $u$ is a constant vector.

Next we consider other classes of Liouville theorems for solutions of (1.1). Recently, Fuchs [Fuc12b] showed that the solution is identical zero under the conditions: $\int_{\mathbf{R}^{2}} h(|\varepsilon(u)|) d x<\infty$ and $\int_{\mathbf{R}^{2}}|u|^{2} d x<\infty$. Now we improve this result and obtain more general types of Liouville theorems.

Before stating Theorem 1.2, we first introduce some notations. If $h$ satisfies the conditions (A1), (A2) and (A3), from [Fuc12b] we know $h^{\prime}(t) \leq C\left(h(t)^{\frac{1}{\tau}}+1\right)$ for some $\tau \in(1,2]$ (see Lemma 2.1 in the Section 2 ). Let $\tau^{\prime}$ be the conjugate exponent of $\tau$ s.t. $\frac{1}{\tau}+\frac{1}{\tau^{\prime}}=1$ and $\tau^{\star}=\frac{\tau^{\prime}-2}{4-\tau^{\prime}}$ provided $2 \leq \tau^{\prime}<4$. For the Navier-Stokes case 
$h(t)=\frac{\nu}{2} t^{2}$, obviously, we can choose $\tau=2$; for general case $h(t)=\mu t^{2}(1+t)^{m}, m>0$, we can choose $1<\tau=\frac{m+2}{m+1}<2$.

Theorem 1.2. Suppose $u \in C^{1}\left(\mathbf{R}^{2}, \mathbf{R}^{2}\right)$ is an entire weak solution of (1.1). Then the following results hold:

(a) If $\frac{3}{2} \leq \tau \leq 2\left(2 \leq \tau^{\prime} \leq 3\right)$ and $u \in L^{p}\left(\mathbf{R}^{2}, \mathbf{R}^{2}\right)$ for some $p>1$, then $u$ is identically zero.

(b) If $\frac{4}{3}<\tau<\frac{3}{2}\left(3<\tau^{\prime}<4\right)$ and $u \in L^{p}\left(\mathbf{R}^{2}, \mathbf{R}^{2}\right)$ for some $p>\tau^{\prime}-2$, then $u$ is identically zero.

(c) If $1<\tau \leq \frac{4}{3}\left(\tau^{\prime} \geq 4\right)$ and $u \in L^{\tau^{\prime}}\left(\mathbf{R}^{2}, \mathbf{R}^{2}\right)$, then $u$ vanishes.

In the setting of the stationary Navier-Stokes equations, $(a)$ clearly implies the following corollary.

Corollary 1.3. Let $u \in C^{1}\left(\mathbf{R}^{2}, \mathbf{R}^{2}\right)$ be an entire weak solution to the stationary Navier-Stokes equations in the plane, i.e.

$$
\int_{\mathbf{R}^{2}} D u: D \varphi d x-\int_{\mathbf{R}^{2}} u^{k} u^{i} \partial_{k} \varphi^{i} d x=0
$$

for all $\varphi \in C_{0}^{\infty}\left(\mathbf{R}^{2}, \mathbf{R}^{2}\right)$ with $\operatorname{div} \varphi=0$, and assume that $u \in L^{p}\left(\mathbf{R}^{2}, \mathbf{R}^{2}\right)$ for some $p>1$. Then $u$ must be the zero vector.

We first comment on the regularity assumption on the solutions. As we know, for general $h$ satisfying (A1)-(A3), $C^{1, \alpha}$ regularity of solutions is an open problem. But in some special case, such as $h(t)=t^{2}(1+t)^{m}, m \geq 0$, the solution $u$ belongs to the space $C^{1, \alpha}$, see e.g. [BFZ05]. For the further discussion about regularity of the solutions of (1.1) the readers are recommended to see the paper [Fuc12a]. Therefore the regularity assumptions on $u$ in the above theorems are reasonable in some sense.

Secondly, we need to say some words about the proof of the theorems. For obtaining Theorem 1.1, in the special case $h(t)=\frac{\nu}{2} t^{2}$, Gilbarg and Weinberger [GW78] used the idea that the vorticity $\omega=\partial_{x_{1}} u^{2}-\partial_{x_{2}} u^{1}$ satisfies the elliptic equation $-\triangle \omega+u \cdot D \omega=0$, which implies that $\omega=0$ by the maximum principle. Furthermore, $u$ is a harmonic field and the Liouville theorem for harmonic functions implies that $\mathrm{u}$ must be a constant vector. But now, due to nonlinearity of the item $\operatorname{div}[T(\varepsilon(u))]$, this approach does not work. In the spirit of [Fuc12b], [FZ12] and [Zha13], we will instead show energy estimates for the second order derivatives from which we deduce that

$$
\int_{\mathbf{R}^{2}} D^{2} H(\varepsilon(u))\left(\varepsilon\left(\partial_{k} u\right), \varepsilon\left(\partial_{k} u\right)\right) d x=0,
$$

which implies that $u$ must be a constant vector under the condition $\int_{\mathbf{R}^{2}} h(|D u|) d x<$ $\infty$.

On the other hand, the strategy for proving 1.2 is as follow: if $p$ is "sufficiently" small, we directly use the local energy estimate for the first order derivatives to control the local integral of $|u|^{q}$ for $q$ large enough. Hence by applying an iteration technique it follows that $\int_{\mathbf{R}^{2}}|u|^{q} d x=0$. If $p$ is large "enough", we will take the derivatives of the equations and use a Caccioppoli-type inequality to obtain a locally finite energy estimate for the second order derivatives, from which, by Sobolev imbedding theorem it follows that $u \in L^{\infty}\left(\mathbf{R}^{2}, \mathbf{R}^{2}\right)$. Thus, in view of the recent result of [Zha13], $u$ must be a constant vector. 
Our notation are standard. Throughout this paper, the convention of summation with respect to indices repeated twice is used. All constants are denoted by the symbol $C$, and $C$ may change from line to line. Whenever it is necessary we will indicate the dependence of $C$ on parameters. As usual $Q_{R}\left(x_{0}\right)$ denotes the open square with center $x_{0}$ and side length $2 R$, and the symbols :, ' will be used for the scalar product of matrices and vectors respectively. $|\cdot|$ denotes the associated Euclidean norms.

Our paper is organized as follows: in Section 2 we present some auxiliary results, in Section 3 we give the proof of Theorem 1.1 and in Section 4 we give the proof of Theorem 1.2.

\section{Auxiliary results}

2.1. Properties of the function $\boldsymbol{h}$. The following properties of the function $h$ follow from (A1)-(A3) in an elementary way, see [Fuc12b]:

(i) $\mu(t)=\frac{h^{\prime}(t)}{t}$ is an increasing function.

(ii) we have $h(0)=h^{\prime}(0)=0$ and

$$
h(t) \geq \frac{1}{2} h^{\prime \prime}(0) t^{2} .
$$

Moreover,

$$
\frac{h^{\prime}(t)}{t} \geq \lim _{s \rightarrow 0} \frac{h^{\prime}(s)}{s}=h^{\prime \prime}(0)>0 .
$$

(iii) The function $h$ satisfies the balancing condition, i.e.

$$
\frac{1}{a} h^{\prime}(t) t \leq h(t) \leq t h^{\prime}(t), \quad t \geq 0 .
$$

(iv) For some exponent $m \geq 2$ and a constant $C \geq 0$ it holds

$$
h(t) \leq C\left(1+t^{m}\right), \quad h^{\prime}(t) \leq C\left(1+t^{m-1}\right), \quad t \geq 0 .
$$

From the assumptions on $h$ and the definition of $H$ stated in (1.2) we deduce the following estimate valid for all $\varepsilon, \sigma \in S^{2}$

$$
\frac{h^{\prime}(|\varepsilon|)}{|\varepsilon|}|\sigma|^{2} \leq D^{2} H(\varepsilon)(\sigma, \sigma) \leq h^{\prime \prime}(|\varepsilon|)|\sigma|^{2},
$$

from which, together with $(2.2)$, it follows that

$$
D^{2} H(\varepsilon)(\sigma, \sigma) \geq h^{\prime \prime}(0)|\sigma|^{2} .
$$

The next lemma is taken from Lemma 2.5 in [Fuc12b].

Lemma 2.1. There is a number $\tau \in(1,2]$ such that

$$
h^{\prime}(t) \leq C\left(h(t)^{\frac{1}{\tau}}+1\right)
$$

or equivalently

$$
|D H(|\varepsilon(u)|)| \leq C\left(H(|\varepsilon(u)|)^{\frac{1}{\tau}}+1\right)
$$

holds for all $t \geq 0$ and $\varepsilon \in S^{2}$. Moreover we even have the sharper estimate

$$
h^{\prime}(t) \leq C\left(h(t)^{\frac{1}{\tau}}+t\right), t \geq 0 .
$$

Remark 2.2. If $h(t)=\frac{\nu}{2} t^{2}$, obviously, we can choose $\tau=2$; for general case $h(t)=\mu t^{2}(1+t)^{m}, m>0$, we can choose $1<\tau=\frac{m+2}{m+1}<2$. 
2.2. Divergence equation and Korn's inequality. First, we recall a standard result concerning the "divergence equation", see e.g. [Gal94a], [Gal94b] or [FS00].

Lemma 2.3. Consider a function $f \in L^{q}\left(Q_{R}(z)\right)$ for some $q>1$ such that $\int_{Q_{R}(z)} f d x=0$. Then there exists a field $v \in W_{0}^{1, q}\left(Q_{R}(z), \mathbf{R}^{2}\right)$ and a constant $C(q)$ independent of $Q_{R}(z)$ such that we have div $v=f$ on $Q_{R}(z)$ together with the estimate

$$
\int_{Q_{R}(z)}|D v|^{q} d x \leq C(q) \int_{Q_{R}(z)}|f|^{q} d x .
$$

We have the following Korn-type inequalities (compare [Tem83]):

Lemma 2.4. There is an absolute constant $C$ such that for all $v \in W_{0}^{1,2}\left(Q_{R}(z)\right.$, $\mathbf{R}^{2}$ ) it holds

$$
\int_{Q_{R}(z)}|D v|^{2} d x \leq C \int_{Q_{R}(z)}|\varepsilon(v)|^{2} d x
$$

and for any $v \in W^{1,2}\left(Q_{R}(z), \mathbf{R}^{2}\right)$ we have

$$
\int_{Q_{R}(z)}|D v|^{2} d x \leq C\left(\int_{Q_{R}(z)}|\varepsilon(v)|^{2} d x+\frac{1}{R^{2}} \int_{Q_{R}(z)}|v|^{2} d x\right) .
$$

2.3. Ladyzhenskaya's inequality and the Sobolev-Poincaré estimate. We start with a version of the Sobolev inequality in the plane, see [GT83].

Lemma 2.5. Let $x_{0} \in \mathbf{R}^{2}, R>0$ and $u \in W^{1,2}\left(Q_{R}\left(x_{0}\right)\right)$. Then, for any $q>1$, there exists a constant $C(q)$ depending only on $q$ such that the following inequality holds

$$
\left(\frac{1}{R^{2}} \int_{Q_{R}\left(x_{0}\right)}|u|^{q} d x\right)^{\frac{1}{q}} \leq C(q)\left\{\left(\int_{Q_{R}\left(x_{0}\right)}|D u|^{2} d x\right)^{\frac{1}{2}}+\left(\frac{1}{R^{2}} \int_{Q_{R}\left(x_{0}\right)}|u|^{2} d x\right)^{\frac{1}{2}}\right\} .
$$

We believe that the following result exists somewhere, because it is an easy consequence of Ladyzhenskaya's inequality. However, it is hard to trace, so we will state and prove it below.

Lemma 2.6. Suppose $v \in W^{1,2}\left(Q_{R}\left(x_{0}\right)\right)$. Then there exists a constant $C_{0}$ independent of $R, x_{0}$ such that

$$
\int_{Q_{R}\left(x_{0}\right)}|v|^{4} d x \leq C_{0}\left\{\int_{Q_{R}\left(x_{0}\right)}|v|^{2} d x \int_{Q_{R}\left(x_{0}\right)}|D v|^{2} d x+\frac{1}{R^{2}}\left(\int_{Q_{R}\left(x_{0}\right)}|v|^{2} d x\right)^{2}\right\} .
$$

Proof. For any $x_{0} \in \mathbf{R}^{2}$ and $u \in W^{1,2}\left(Q_{1}\left(x_{0}\right)\right)$, there exists an extension $\tilde{u} \in$ $W_{0}^{1,2}\left(Q_{2}\left(x_{0}\right)\right)$ of $u$ s.t.

$$
\|\tilde{u}\|_{L^{2}\left(Q_{2}\left(x_{0}\right)\right)} \leq C\|u\|_{L^{2}\left(Q_{1}\left(x_{0}\right)\right)}
$$

and

$$
\|\tilde{u}\|_{W_{0}^{1,2}\left(Q_{2}\left(x_{0}\right)\right)} \leq C\|u\|_{W^{1,2}\left(Q_{1}\left(x_{0}\right)\right)},
$$

where $C$ is an absolute constant. We refer the reader to [Eva98].

Moreover, by Ladyzhenskaya's inequality (see [Lad69], Lemma 1 on p. 8) we have

$$
\int_{Q_{2}\left(x_{0}\right)}|\tilde{u}|^{4} d x \leq 2 \int_{Q_{2}\left(x_{0}\right)}|\tilde{u}|^{2} d x \int_{Q_{2}\left(x_{0}\right)}|D \tilde{u}|^{2} d x .
$$


Putting together the estimates (2.7), (2.8) and (2.9) we deduce

$$
\begin{aligned}
\int_{Q_{2}\left(x_{0}\right)}|\tilde{u}|^{4} d x & \leq C\left(\int_{Q_{1}\left(x_{0}\right)}|D u|^{2} d x+\int_{Q_{1}\left(x_{0}\right)}|u|^{2} d x\right) \int_{Q_{1}\left(x_{0}\right)}|u|^{2} d x \\
& \leq C\left\{\int_{Q_{1}\left(x_{0}\right)}|u|^{2} d x \int_{Q_{1}\left(x_{0}\right)}|D u|^{2} d x+\left(\int_{Q_{1}\left(x_{0}\right)}|u|^{2} d x\right)^{2}\right\},
\end{aligned}
$$

from which it follows that

$$
\int_{Q_{1}\left(x_{0}\right)}|u|^{4} d x \leq C\left\{\int_{Q_{1}\left(x_{0}\right)}|u|^{2} d x \int_{Q_{1}\left(x_{0}\right)}|D u|^{2} d x+\left(\int_{Q_{1}\left(x_{0}\right)}|u|^{2} d x\right)^{2}\right\} .
$$

Now, if $u(x) \in W^{1,2}\left(Q_{R}\left(x_{0}\right)\right)$ and $v(x):=u(R x)$, then $v \in W^{1,2}\left(Q_{1}\left(x_{0}\right)\right)$. Applying (2.10) and recalling the change of variable formula we end up with

$$
\int_{Q_{R}\left(x_{0}\right)}|u|^{4} d x \leq C\left\{\int_{Q_{R}\left(x_{0}\right)}|u|^{2} d x \int_{Q_{R}\left(x_{0}\right)}|D u|^{2} d x+\frac{1}{R^{2}}\left(\int_{Q_{R}\left(x_{0}\right)}|u|^{2} d x\right)^{2}\right\} .
$$

2.4. A lemma of Gilbarg and Weinberger. The following result is due to Gilbarg and Weinberger, see Lemma 2.1 in [GW78].

Lemma 2.7. Let $f \in C^{1}$ in $\left\{x \in \mathbf{R}^{2}|| x \mid=r>r_{0}>0\right\}$ with finite Dirichlet integral in the sense that

$$
\int_{r>r_{0}}|D f|^{2} d x d y<\infty
$$

Then

$$
\lim _{r \rightarrow \infty} \frac{1}{\log r} \int_{0}^{2 \pi} f(r, \theta)^{2} d \theta=0 .
$$

2.5. A lemma of Giaquinta and Modica. The following $\varepsilon$-lemma goes back to the work of Giaquinta and Modica [GM82]. Recently, Fuchs and the author proved some generalizations of the $\varepsilon$-lemma in [FZ12]. For proving our results, we need the following more general type of the $\varepsilon$-lemma.

Lemma 2.8. Let $f, f_{1}, \ldots, f_{l}$ denote non-negative functions from the space $L_{l o c}^{1}\left(\mathbf{R}^{2}\right)$. Suppose further that we are given exponents $\alpha_{1}, \ldots, \alpha_{l}>0, \beta_{1}, \ldots, \beta_{l}>1$. Suppose that, for any $x_{0} \in \mathbf{R}^{2}, Q=Q_{2 R}\left(x_{0}\right)$, we can find $\delta>0$ depending on $\alpha_{1}, \ldots, \alpha_{l}>0$ such that

$$
\int_{Q_{r}(z)} f d x \leq \delta \int_{Q_{2 r}(z)} f d x+C(\delta) \sum_{j=1}^{l} r^{-\alpha_{j}}\left(\int_{Q_{2 r}(z)} f_{j} d x\right)^{\beta_{j}}
$$

holds for any choice $Q_{2 r}(z) \subset Q_{2 R}\left(x_{0}\right)$. Then there is a constant $C$ independent of $\delta$ and $R$ with the property

$$
\int_{Q_{R}\left(x_{0}\right)} f d x \leq C \sum_{j=1}^{l} R^{-\alpha_{j}}\left(\int_{Q_{2 R}\left(x_{0}\right)} f_{j} d x\right)^{\beta_{j}}
$$

Remark 2.9. The proof of Lemma 2.8 is almost the same as that of the corresponding statement in the appendix of [FZ12] with the slight difference that $\int_{Q_{2 r}(z)} f_{j}$ $d x$ has to be replaced by $\left(\int_{Q_{2 r}(z)} f_{j} d x\right)^{\beta_{j}}$. For overcoming this minor difficulty, we 
just need to notice the validity of the estimate

$$
\left(\int_{Q_{2 r}(z)} f_{j} d x\right)^{\beta_{j}} \leq\left(\int_{Q_{2 R}\left(x_{0}\right)} f_{j} d x\right)^{\beta_{j}-1} \int_{Q_{2 r}(z)} f_{j} d x .
$$

2.6. A Liouville theorem. We recall the following Liouville theorem for solutions of (1.1) established in [Zha13], which plays an important role in proving Theorem 1.2.

Lemma 2.10. Suppose that $u \in C^{1}\left(\mathbf{R}^{2}, \mathbf{R}^{2}\right) \bigcap L^{\infty}\left(\mathbf{R}^{2}, \mathbf{R}^{2}\right)$ is an entire weak solution of (1.1). Then $u$ is a constant vector.

\section{Proof of Theorem 1.1}

In view of $u \in C^{1}\left(\mathbf{R}^{2}, \mathbf{R}^{2}\right)$ and with the help of the ellipticity estimate (2.5), by standard difference quotient technique we have that $u \in W_{\text {loc }}^{2,2}\left(\mathbf{R}^{2}, \mathbf{R}^{2}\right)$, see [Fuc12b], [Zha13]. The proof of Theorem 1.1 is divided into the following three lemmas.

Lemma 3.1. Let $u \in C^{1}\left(\mathbf{R}^{2}, \mathbf{R}^{2}\right)$ be an entire weak solution of (1.1) satisfying the condition $\int_{\mathbf{R}^{2}} h(|D u|) d x<\infty$. Then, for any $x_{0} \in \mathbf{R}^{2}, R>0$, the following energy estimate of the solution holds

$$
\begin{aligned}
\int_{Q_{R}\left(x_{0}\right)} W d x \leq & C\left\{\frac{1}{R^{2}} \int_{Q_{2 R}\left(x_{0}\right)} h(|\varepsilon(u)|) d x+\frac{1}{R^{2}} \int_{Q_{2 R}\left(x_{0}\right)}|D u|^{2} d x\right. \\
& \left.+\left(1+\frac{1}{R^{2 m}}\right)+\frac{1}{R^{3}} \int_{Q_{2 R}\left(x_{0}\right)}|u| d x\right\}
\end{aligned}
$$

where $W=D^{2} H(\varepsilon(u))\left(\varepsilon\left(\partial_{k} u\right), \varepsilon\left(\partial_{k} u\right)\right), m>0$ denotes a suitable positive number defined by the condition (2.4), $C$ is a constant independent of $x_{0}, R$.

Proof. For any cut-off function $\eta \in C_{0}^{\infty}\left(\mathbf{R}^{2}\right)$ with $0 \leq \eta \leq 1$, the following estimate is obtained in [Zha13](see (3.9) in this reference)

$$
\begin{aligned}
& \int_{\mathbf{R}^{2}} D^{2} H(\varepsilon(u))\left(\varepsilon\left(\partial_{k} u\right), \varepsilon\left(\partial_{k} u\right)\right) \eta^{2} d x \\
& \leq C\left\{\int_{\mathbf{R}^{2}} h(|\varepsilon(u)|)|D \eta|^{2} d x+\int_{\mathbf{R}^{2}} h^{\prime}(|\varepsilon(u)|)^{2}\left(|D \eta|^{2}+\left|D^{2} \eta\right|\right) d x\right. \\
& \left.\quad+\int_{\mathbf{R}^{2}}|D u|^{2}\left(|D \eta|^{2}+\left|D^{2} \eta\right|\right) d x+\int_{\mathbf{R}^{2}}|D u|^{2}|u||D \eta| d x\right\} .
\end{aligned}
$$

Now, for any $x \in Q_{2 R}\left(x_{0}\right), r>0$ such that $Q_{2 r}(x) \subset Q_{2 R}\left(x_{0}\right)$ and $\eta \in C_{0}^{\infty}\left(Q_{\frac{3}{2} r}(x)\right)$ satisfying $\eta=1$ in $Q_{r}(x)$ and $0 \leq \eta \leq 1,|D \eta| \leq \frac{4}{r},\left|D^{2} \eta\right| \leq \frac{16}{r^{2}}$, we deduce from (3.2) that

$$
\begin{aligned}
\int_{Q_{r}(x)} W d x \leq C & \left\{\frac{1}{r^{2}} \int_{Q_{\frac{3}{2} r}(x)} h(|\varepsilon(u)|) d x+\frac{1}{r^{2}} \int_{Q_{\frac{3}{2} r}(x)} h^{\prime}(|\varepsilon(u)|)^{2} d x\right. \\
& \left.+\frac{1}{r^{2}} \int_{Q_{\frac{3}{2} r}(x)}|D u|^{2} d x+\frac{1}{r} \int_{T_{\frac{3}{2} r}(x)}|D u|^{2}|u| d x\right\},
\end{aligned}
$$

where $T_{\frac{3}{2} r}(x)=Q_{\frac{3}{2} r}(x) \backslash \overline{Q_{r}(x)}$. 
For the term $\frac{1}{r^{2}} \int_{Q_{\frac{3}{2} r}(x)} h^{\prime}(|\varepsilon(u)|)^{2} d x$, the following estimate has been obtained in formula (3.16) of [Zha13], where $L$ denotes some positive number:

$$
\begin{aligned}
\frac{1}{r^{2}} \int_{Q_{\frac{3}{2} r}(x)} h^{\prime}(|\varepsilon(u)|)^{2} d x \leq & C h^{\prime}(L)^{2}+C \frac{1}{L^{2}} \frac{1}{r^{4}}\left(\int_{Q_{2 r}(x)} h(|\varepsilon(u)|) d x\right)^{2} \\
& +C \frac{1}{r^{2}} \frac{1}{L^{2}} \int_{Q_{2 r}(x)} h(|\varepsilon(u)|) d x \int_{Q_{2 r}(x)} W d x .
\end{aligned}
$$

Choosing $L=\frac{1}{\varepsilon^{\frac{1}{2}} r}, \varepsilon<1$, we deduce from (3.4) that

$$
\begin{aligned}
\frac{1}{r^{2}} \int_{Q_{\frac{3}{2} r}(x)} h^{\prime}(|\varepsilon(u)|)^{2} d x \leq & C h^{\prime}\left(\frac{1}{\varepsilon^{\frac{1}{2}} r}\right)^{2}+C \frac{1}{r^{2}}\left(\int_{Q_{2 r}(x)} h(|\varepsilon(u)|) d x\right)^{2} \\
& +C \varepsilon \int_{Q_{2 r}(x)} h(|\varepsilon(u)|) d x \int_{Q_{2 r}(x)} W d x
\end{aligned}
$$

It remains to discuss the term $\frac{1}{r} \int_{T_{\frac{3}{2} r}(x)}|u||D u|^{2} d x$. Letting $A=f_{T_{\frac{3}{2} r}(x)}|D u|^{2} d x$ and $B=f_{T_{\frac{3}{2} r}(x)} u d x$, we have

$$
\begin{aligned}
\frac{1}{r} \int_{T_{\frac{3}{2} r}(x)}|D u|^{2}|u| d x \leq & \left.\frac{1}{r} \int_{T_{\frac{3}{2} r}(x)}|| D u\right|^{2}-A|| u-B \mid d x \\
& +\frac{1}{r}|B| \int_{T_{\frac{3}{2} r}(x)}|D u|^{2} d x+\frac{1}{r}|A| \int_{T_{\frac{3}{2} r}(x)}|u-B| d x .
\end{aligned}
$$

Using the definitions of $A, B$ and applying Young's inequality in (3.6) we obtain, for $\varepsilon>0$,

$$
\begin{aligned}
\frac{1}{r} \int_{T_{\frac{3}{2} r}(x)}|D u|^{2}|u| d x \leq & \left.\varepsilon \int_{T_{\frac{3}{2} r}(x)}|| D u\right|^{2}-\left.A\right|^{2} d x+\frac{1}{\varepsilon} \frac{1}{r^{2}} \int_{T_{\frac{3}{2} r}(x)}|u-B|^{2} d x \\
& +\frac{C}{r^{3}} \int_{T_{\frac{3}{2} r}(x)}|D u|^{2} d x \int_{T_{\frac{3}{2} r}(x)}|u| d x .
\end{aligned}
$$

Applying Poincaré's inequality in (3.7) we find

$$
\begin{aligned}
\frac{1}{r} \int_{T_{\frac{3}{2} r}(x)}|D u|^{2}|u| d x \leq & \varepsilon\left(\int_{T_{\frac{3}{2} r}(x)} D\left(|D u|^{2}\right) d x\right)^{2}+\frac{1}{\varepsilon} \int_{T_{\frac{3}{2} r}(x)}|D u|^{2} d x \\
& +\frac{C}{r^{3}} \int_{T_{\frac{3}{2} r}(x)}|D u|^{2} d x \int_{T_{\frac{3}{2} r}(x)}|u| d x
\end{aligned}
$$

from which, together with Hölder's inequality, it follows that

$$
\begin{aligned}
\frac{1}{r} \int_{T_{\frac{3}{2} r}(x)}|D u|^{2}|u| d x & \leq \varepsilon \int_{T_{\frac{3}{2} r}(x)}|D u|^{2} d x \int_{T_{\frac{3}{2} r}(x)}\left|D^{2} u\right|^{2} d x \\
& +\frac{1}{\varepsilon} \int_{T_{\frac{3}{2} r}(x)}|D u|^{2} d x+\frac{C}{r^{3}} \int_{T_{\frac{3}{2} r}(x)}|D u|^{2} d x \int_{T_{\frac{3}{2} r}(x)}|u| d x .
\end{aligned}
$$


Combining (3.3), (3.5) and (3.9) and observing the inequality $\left|D^{2} u(x)\right| \leq C|D \varepsilon(x)|$ $\leq C W(x)$ we deduce that

$$
\begin{aligned}
& \int_{Q_{r}(x)} W d x \\
& \leq C \varepsilon \int_{T_{\frac{3}{2} r}(x)}|D u|^{2} d x \int_{Q_{2 r}(x)} W d x+C \varepsilon \int_{Q_{2 r}(x)} h(|\varepsilon(u)|) d x \int_{Q_{2 r}(x)} W d x \\
& \quad+\frac{C}{r^{2}} \int_{Q_{2 r}(x)} h(|\varepsilon(u)|) d x+\frac{C}{r^{2}} \int_{Q_{2 r}(x)}|D u|^{2} d x+C h^{\prime}\left(\frac{1}{\varepsilon^{\frac{1}{2}} r}\right)^{2} \\
& \quad+\frac{C}{r^{2}}\left(\int_{Q_{2 r}(x)} h(|\varepsilon(u)|) d x\right)^{2}+\frac{C}{\varepsilon} \int_{T_{\frac{3}{2} r}(x)}|D u|^{2} d x \\
& \quad+\frac{C}{r^{3}} \int_{T_{\frac{3}{2} r}(x)}|D u|^{2} d x \int_{T_{\frac{3}{2} r}(x)}|u| d x .
\end{aligned}
$$

Since $\int_{\mathbf{R}^{2}}|D u|^{2} d x \leq C \int_{\mathbf{R}^{2}} h(|D u|) d x<\infty$, choosing $\varepsilon$ small enough and letting $\delta:=C \varepsilon<\frac{1}{2}$ we find from (3.10) that

$$
\begin{aligned}
\int_{Q_{r}(x)} W d x \leq & \delta \int_{Q_{2 r}(x)} W d x+\frac{C}{r^{2}} \int_{Q_{2 r}(x)} h(|\varepsilon(u)|) d x+\frac{C}{r^{2}} \int_{Q_{2 r}(x)}|D u|^{2} d x \\
& +C \int_{T_{\frac{3}{2} r}(x)}|D u|^{2} d x+C h^{\prime}\left(\frac{1}{\varepsilon^{\frac{1}{2}} r}\right)^{2}+\frac{C}{r^{3}} \int_{Q_{\frac{3}{2} r}(x)}|u| d x .
\end{aligned}
$$

In view of the condition (2.4) and on account of the inequality $\int_{\mathbf{R}^{2}}|D u|^{2} d x \leq$ $C \int_{\mathbf{R}^{2}} h(|D u|) d x<\infty$ it follows that

$$
\begin{aligned}
\int_{Q_{r}(x)} W d x \leq & \delta \int_{Q_{2 r}(x)} W d x+\frac{C}{r^{2}} \int_{Q_{2 r}(x)} h(|\varepsilon(u)|) d x+\frac{C}{r^{2}} \int_{Q_{2 r}(x)}|D u|^{2} d x \\
& +C\left(1+\frac{1}{r^{m}}\right)+\frac{C}{r^{3}} \int_{Q_{2 r}(x)}|u| d x .
\end{aligned}
$$

By Lemma 2.6 we end up with

$$
\begin{aligned}
\int_{Q_{R}\left(x_{0}\right)} W d x \leq & C\left\{\frac{1}{R^{2}} \int_{Q_{2 R}\left(x_{0}\right)} h(|\varepsilon(u)|) d x+\frac{1}{R^{2}} \int_{Q_{2 R}\left(x_{0}\right)}|D u|^{2} d x\right. \\
& \left.+\left(1+\frac{1}{R^{m}}\right)+\frac{1}{R^{3}} \int_{Q_{2 R}\left(x_{0}\right)}|u| d x\right\},
\end{aligned}
$$

and (3.13) corresponds to our claim (3.1).

Lemma 3.2. Let $u$ satisfy the same conditions as stated in Lemma 3.1. Then the following estimate holds

$$
\int_{\mathbf{R}^{2}} D^{2} H(\varepsilon(u))\left(\varepsilon\left(\partial_{k} u\right), \varepsilon\left(\partial_{k} u\right)\right) d x<\infty
$$

and in conclusion

$$
\int_{\mathbf{R}^{2}}\left|D^{2} u\right|^{2} d x<\infty
$$


Proof. Since $\int_{\mathbf{R}^{2}} h(|D u|) d x=: M<\infty$, for $R>1$, (3.13) gives

$$
\int_{Q_{R}\left(x_{0}\right)} W d x \leq C(M)+\frac{C}{R^{3}} \int_{Q_{2 R}\left(x_{0}\right)}|u| d x .
$$

Since $u \in C^{1}\left(\mathbf{R}^{2}, \mathbf{R}^{2}\right)$ and $\int_{\mathbf{R}^{2}}|D u|^{2} d x \leq C \int_{\mathbf{R}^{2}} h(|D u|) d x<\infty$, using Lemma 2.3 we deduce that

$$
\limsup _{R \rightarrow \infty} \frac{1}{R^{3}} \int_{Q_{2 R}\left(x_{0}\right)}|u| d x=0
$$

Letting $R \rightarrow \infty$ in (3.16) we have

$$
\int_{\mathbf{R}^{2}} W d x<\infty
$$

Since $\left|D^{2} u(x)\right| \leq C|D \varepsilon(u)(x)|,(3.18)$ implies (3.15). Thus, the proof is complete.

Lemma 3.3. Let $u$ satisfy the same conditions as stated in Lemma 3.1. Then we have

$$
\int_{\mathbf{R}^{2}} W d x=0
$$

hence, $u$ must be a constant vector.

Proof. From $\int_{\mathbf{R}^{2}}|D u|^{2} d x \leq C \int_{\mathbf{R}^{2}} h(|D u|) d x<\infty$, it follows that $\lim _{r \rightarrow \infty} \int_{T_{\frac{3}{2} r}(x)}$ $|D u|^{2} d x=0$. In view of the condition $h^{\prime}(0)=0$ and (3.17), taking $x=0$ in (3.11) and letting $r \rightarrow \infty$ in (3.11) we have

$$
\int_{\mathbf{R}^{2}} W+, d x \leq \frac{1}{2} \int_{\mathbf{R}^{2}} W d x
$$

from which, together with the estimate (3.18), it follows that

$$
\int_{\mathbf{R}^{2}} W d x=0
$$

Obviously, (3.22) implies $W(x)=0$. From the relation $\left|D^{2} u(x)\right|^{2} \leq C|D \varepsilon(x)|^{2} \leq$ $C W(x)$, we know that $D^{2} u(x)=0$. Therefore $u$ must be an affine function. On the other hand, in view of the inequality $\int_{\mathbf{R}^{2}}|D u|^{2} d x \leq C \int_{\mathbf{R}^{2}} h(|D u|) d x<\infty$, we obtain $D u(x)=0$, thus, $u$ is a constant vector.

\section{Proof of 1.2}

Under the integrability condition of $|u|^{p}$, for obtaining a Liouville type theorem for the solutions $u$ of (1.1), we should verify that the first order derivatives energy quantity as well as the second order derivatives energy term are controlled by the integral of $|u|^{p}$. The details are stated below and the proof of 1.2 is broken into the following four lemmas. 
Lemma 4.1. Let $u \in C^{1}\left(\mathbf{R}^{2}, \mathbf{R}^{2}\right)$ be an entire weak solution of (1.1). Then, for any $x_{0} \in \mathbf{R}^{2}, R>0$, the following energy estimate holds

$$
\begin{aligned}
\int_{Q_{R}\left(x_{0}\right)} h(|\varepsilon(u)|) d x \leq & C\left\{\frac{1}{R^{\tau^{\prime}}} \int_{Q_{2 R}\left(x_{0}\right)}|u|^{\tau^{\prime}} d x+\frac{1}{R^{2}} \int_{Q_{2 R}\left(x_{0}\right)}|u|^{2} d x\right. \\
& \left.+\frac{1}{R^{2}}\left(\int_{Q_{2 R}\left(x_{0}\right)}|u|^{2} d x\right)^{2}\right\} .
\end{aligned}
$$

Proof. For any $x_{0} \in \mathbf{R}^{2}, R>0$ and $x \in Q_{2 R}\left(x_{0}\right), r>0$ s.t. $Q_{2 r}(x) \subset Q_{2 R}\left(x_{0}\right)$, we choose the cut-off function $\eta \in C_{0}^{\infty}\left(Q_{\frac{3}{2} r}(x)\right)$ as done in the proof of Lemma 3.1 and find a solution $\varpi$ to the following equation

$$
\operatorname{div} \varpi=\operatorname{div}\left(u \eta^{2}\right)=u \cdot D \eta^{2} \quad \text { on } Q_{2 r}(x)
$$

s.t.

$$
\operatorname{spt} \varpi \subset Q_{\frac{3}{2} r}(x)
$$

and for any $q>1$, the following estimate holds

$$
\|\varpi\|_{W^{1, q}\left(Q_{\frac{3}{2} r}(x)\right)} \leq C(q)\left\|u \cdot D \eta^{2}\right\|_{L^{q}\left(Q_{\frac{3}{2} r}(x)\right)} .
$$

Taking the test function $\varphi=u \eta^{2}-\varpi$ in (1.1) we obtain

$$
\begin{gathered}
\int_{\mathbf{R}^{2}} D H(\varepsilon(u)): \varepsilon\left(u \eta^{2}\right) d x-\int_{\mathbf{R}^{2}} D H(\varepsilon(u)): \varepsilon(\varpi) d x-\int_{\mathbf{R}^{2}} u^{i} u^{j} \\
\partial_{i}\left(u^{j} \eta^{2}\right) d x+\int_{\mathbf{R}^{2}} u^{i} u^{j} \partial_{i} \varpi^{j} d x=0 .
\end{gathered}
$$

Hence,

$$
\begin{aligned}
\int_{\mathbf{R}^{2}} D H(\varepsilon(u)): \varepsilon(u) \eta^{2} d x= & -\int_{\mathbf{R}^{2}} D H(\varepsilon(u)): u \otimes D \eta^{2} d x+\int_{\mathbf{R}^{2}} D H(\varepsilon(u)) \\
& : \varepsilon(\varpi) d x+\int_{\mathbf{R}^{2}} u^{i} u^{j} \partial_{i}\left(u^{j} \eta^{2}\right) d x-\int_{\mathbf{R}^{2}} u^{i} u^{j} \partial_{i} \varpi^{j} d x \\
= & : I+I I+I I I+I V .
\end{aligned}
$$

Recalling the definition of $H$ and the properties of $\eta$ and applying Lemma 2.4 and Young's inequality, for any $0<\delta<1$, we have

$$
\begin{aligned}
I & \leq \int_{\mathbf{R}^{2}} h^{\prime}(|\varepsilon(u)|)|u||D \eta| d x \leq C \int_{\mathbf{R}^{2}}\left(h(|\varepsilon(u)|)^{\frac{1}{\tau}}+|\varepsilon(u)|\right)|u||D \eta| d x \\
& \leq \delta \int_{Q_{\frac{3}{2} r}(x)} h(|\varepsilon(u)|) d x+C(\tau, \delta)\left\{\frac{1}{r^{2}} \int_{Q_{\frac{3}{2} r}(x)}|u|^{2} d x+\frac{1}{r^{\tau^{\prime}}} \int_{Q_{\frac{3}{2} r}(x)}|u|^{\tau^{\prime}} d x\right\} .
\end{aligned}
$$

Using the same way to deal with $I I$, we obtain

$$
\begin{gathered}
I I \leq \delta \int_{Q_{\frac{3}{2} r}(x)} h(|\varepsilon(u)|) d x+C(\tau, \delta)\left\{\frac{1}{r^{2}} \int_{Q_{\frac{3}{2} r}(x)}|\varepsilon(\varpi)|^{2} d x\right. \\
\left.+\frac{1}{r^{\tau^{\prime}}} \int_{Q_{\frac{3}{2} r}(x)}|\varepsilon(\varpi)|^{\tau^{\prime}} d x\right\} .
\end{gathered}
$$


Putting together the estimates (4.4) and (4.8) we find

$$
I I \leq \delta \int_{Q_{\frac{3}{2} r}(x)} h(|\varepsilon(u)|) d x+C(\tau, \delta)\left\{\frac{1}{r^{2}} \int_{Q_{\frac{3}{2} r}(x)}|u|^{2} d x+\frac{1}{r^{\tau^{\prime}}} \int_{Q_{\frac{3}{2} r}(x)}|u|^{\tau^{\prime}} d x\right\}
$$

For $I I I$, using the condition divu $=0$ and Young's inequality we deduce by integration by parts that, for any $\varepsilon>0$,

$$
\begin{aligned}
I I I & =\int_{\mathbf{R}^{2}} u^{i} u^{j} \partial_{i}\left(u^{j} \eta^{2}\right) d x=\int_{\mathbf{R}^{2}} u^{i} u^{j} \partial_{i} u^{j} \eta^{2} d x+\int_{\mathbf{R}^{2}} u^{i} u^{j} u^{j} \partial_{i} \eta^{2} d x \\
& =\int_{\mathbf{R}^{2}} \frac{|u|^{2}}{2} u \cdot D \eta^{2} \leq \frac{C}{r} \int_{Q_{\frac{3}{2} r}(x)}|u|^{3} d x \\
& \leq \varepsilon \int_{Q_{\frac{3}{2} r}(x)}|u|^{4} d x+\frac{C}{\varepsilon r^{2}} \int_{Q_{\frac{3}{2} r}(x)}|u|^{2} d x .
\end{aligned}
$$

It remains to deal with $I V$. By Young's inequality and (4.4) we have

$$
I V \leq \varepsilon \int_{Q_{\frac{3}{2} r}(x)}|u|^{4} d x+\frac{C}{\varepsilon r^{2}} \int_{Q_{\frac{3}{2} r}(x)}|u|^{2} d x
$$

Furthermore, from Lemma 2.5 it follows that

$$
\int_{Q_{\frac{3}{2} r}(x)}|u|^{4} d x \leq C_{0} \int_{Q_{\frac{3}{2} r}(x)}|u|^{2} d x \int_{Q_{\frac{3}{2} r}(x)}|D u|^{2} d x+\frac{C_{0}}{r^{2}}\left(\int_{Q_{\frac{3}{2} r}(x)}|u|^{2} d x\right)^{2} .
$$

Choosing $\varepsilon=\frac{\delta}{C_{0}\left(1+\int_{Q_{\frac{3}{2} r}(x)}|u|^{2} d x\right)}$, we have the following valid estimate for $I I I$ and $I V$,

$$
\begin{aligned}
& I I I, I V \\
& \leq \delta \int_{Q_{\frac{3}{2} r}(x)}|D u|^{2} d x+C(\delta)\left\{\frac{1}{r^{2}} \int_{Q_{\frac{3}{2} r}(x)}|u|^{2} d x+\frac{1}{r^{2}}\left(\int_{Q_{\frac{3}{2} r}(x)}|u|^{2} d x\right)^{2}\right\} .
\end{aligned}
$$

Now, we want to give an appropriate control for the term $\int_{Q_{\frac{3}{2} r}(x)}|D u|^{2} d x$. Choosing the cut-off function $\xi$ s.t. $\xi \in C_{0}^{\infty}\left(Q_{2 r}(x)\right), 0 \leq \xi \leq 1, \xi=1$ on $Q_{\frac{3}{2} r}(x)$ and $|D \xi| \leq \frac{4}{r}$, we deduce from Lemma 2.2 that

$$
\begin{aligned}
\int_{Q_{\frac{3}{2} r}(x)}|D u|^{2} d x & \leq \int_{Q_{2 r}(x)}|D(u \xi)|^{2} d x \leq C \int_{Q_{2 r}(x)}|\varepsilon(u \xi)|^{2} d x \\
& \leq C \int_{Q_{2 r}(x)}|\varepsilon(u)|^{2} d x+\frac{C}{r^{2}} \int_{Q_{2 r}(x)}|u|^{2} d x
\end{aligned}
$$


Putting together the estimates (4.6), (4.7), (4.9), (4.13) and (4.14) and using the relation $h(\varepsilon(u)) \geq \frac{1}{2} h^{\prime \prime}(0)|\varepsilon(u)|^{2}, h^{\prime \prime}(0)>0$ we have

$$
\begin{aligned}
& \int_{\mathbf{R}^{2}} D H(\varepsilon(u)): \varepsilon(u) \eta^{2} d x \leq C \delta \int_{Q_{2 r}(x)} h(|\varepsilon(u)|) d x \\
& +C(\delta, \tau)\left\{\frac{1}{r^{\tau^{\prime}}} \int_{Q_{\frac{3}{2} r}(x)}|u|^{\tau^{\prime}} d x+\frac{1}{r^{2}} \int_{Q_{2 r}(x)}|u|^{2} d x+\frac{1}{r^{2}}\left(\int_{Q_{2 r}(x)}|u|^{2} d x\right)^{2}\right\} .
\end{aligned}
$$

Recalling the definition of $\eta$ and the condition (2.3) and choosing $\delta$ small enough we obtain

$$
\begin{aligned}
\int_{Q_{r}(x)} h(|\varepsilon(u)|) d x \leq & C \delta \int_{Q_{2 r}(x)} h(|\varepsilon(u)|) d x+C(\tau)\left\{\frac{1}{r^{\tau^{\prime}}} \int_{Q_{2 r}(x)}|u|^{\tau^{\prime}} d x\right. \\
& \left.+\frac{1}{r^{2}} \int_{Q_{2 r}(x)}|u|^{2} d x+\frac{1}{r^{2}}\left(\int_{Q_{2 r}(x)}|u|^{2} d x\right)^{2}\right\},
\end{aligned}
$$

from which, together with Lemma 2.6, it follows that

$$
\begin{aligned}
\int_{Q_{R}\left(x_{0}\right)} h(|\varepsilon(u)|) d x \leq & C\left\{\frac{1}{R^{\tau^{\prime}}} \int_{Q_{2 R}\left(x_{0}\right)}|u|^{\tau^{\prime}} d x+\frac{1}{R^{2}} \int_{Q_{2 R}\left(x_{0}\right)}|u|^{2} d x\right. \\
& \left.+\frac{1}{R^{2}}\left(\int_{Q_{2 R}\left(x_{0}\right)}|u|^{2} d x\right)^{2}\right\}
\end{aligned}
$$

and (4.1) is established.

Lemma 4.2. Let $u \in C^{1}\left(\mathbf{R}^{2}, \mathbf{R}^{2}\right)$ be an entire weak solution of (1.1) and assume that $2 \leq \tau^{\prime}<4$. Then, for any $x_{0} \in \mathbf{R}^{2}, R>0$ we have

$$
\begin{aligned}
\int_{Q_{R}\left(x_{0}\right)} h(|\varepsilon(u)|) d x \leq & C(\tau)\left\{\frac{1}{R^{2}} \int_{Q_{2 R}\left(x_{0}\right)}|u|^{2} d x+\frac{1}{R^{\bar{\tau}}} \int_{Q_{2 R}\left(x_{0}\right)}|u|^{2} d x\right. \\
& \left.+\frac{1}{R^{2}}\left(\int_{Q_{2 R}\left(x_{0}\right)}|u|^{2} d x\right)^{2}+\frac{1}{R^{\bar{\tau}}}\left(\int_{Q_{2 R}\left(x_{0}\right)}|u|^{2} d x\right)^{\tau^{\star}+1}\right\}
\end{aligned}
$$

where $\bar{\tau}=\frac{2 \tau^{\prime}}{4-\tau^{\prime}} \geq 2$ and $\tau^{\star}=\frac{\tau^{\prime}-2}{4-\tau^{\prime}}$.

Proof. Returning to (4.15), we just need to control $\left.\frac{1}{r^{\tau^{\prime}}} \int_{Q_{\frac{3}{2} r}(x)}|u|\right|^{\tau^{\prime}} d x$. By Young's inequality we have, for any $\varepsilon>0$,

$$
\frac{1}{r^{\tau^{\prime}}} \int_{Q_{\frac{3}{2} r}(x)}|u|^{\tau^{\prime}} d x \leq \varepsilon \int_{Q_{\frac{3}{2} r}(x)}|u|^{4} d x+C(\tau) \frac{1}{\varepsilon^{\tau^{\star}}} \frac{1}{r^{\bar{\tau}}} \int_{Q_{\frac{3}{2} r}(x)}|u|^{2} d x .
$$

Moreover, by Lemma 2.5 we find

$$
\int_{Q_{\frac{3}{2} r}(x)}|u|^{4} d x \leq C_{0} \int_{Q_{\frac{3}{2} r}(x)}|u|^{2} d x \int_{Q_{\frac{3}{2} r}(x)}|D u|^{2} d x+C_{0} \frac{1}{r^{2}}\left(\int_{Q_{\frac{3}{2} r}(x)}|u|^{2} d x\right)^{2} .
$$


Thus, putting together the estimates (4.17), (4.18) we have

$$
\begin{aligned}
\frac{1}{r^{\tau^{\prime}}} \int_{Q_{\frac{3}{2} r}(x)}|u|^{\tau^{\prime}} d x \leq & \varepsilon C_{0} \int_{Q_{\frac{3}{2} r}(x)}|u|^{2} d x \int_{Q_{\frac{3}{2} r}(x)}|D u|^{2} d x \\
& +\varepsilon C_{0} \frac{1}{r^{2}}\left(\int_{Q_{\frac{3}{2} r}(x)}|u|^{2} d x\right)^{2}+C(\tau) \frac{1}{\varepsilon^{\tau^{\star}}} \frac{1}{r^{\bar{\tau}}} \int_{Q_{\frac{3}{2} r}(x)}|u|^{2} d x .
\end{aligned}
$$

Letting $\varepsilon=\frac{\delta}{C_{0}\left(1+\int_{Q_{\frac{3}{2}}(x)}|u|^{2} d x\right)}$ and combining the estimates (4.15), (4.19), in view of $\eta=1$ in $Q_{r}(x)$, we end up with

$$
\begin{aligned}
\int_{Q_{r}(x)} h(|\varepsilon(u)|) d x \leq & C \delta \int_{Q_{2 r}(x)} h(|\varepsilon(u)|) d x+\delta \int_{Q_{\frac{3}{2} r}(x)}|D u|^{2} d x \\
& +C(\tau, \delta)\left\{\frac{1}{r^{2}} \int_{Q_{2 r}(x)}|u|^{2} d x+\frac{1}{r^{\bar{\tau}}} \int_{Q_{2 r}(x)}|u|^{2} d x\right. \\
& \left.+\frac{1}{r^{2}}\left(\int_{Q_{2 r}(x)}|u|^{2} d x\right)^{2}+\frac{1}{r^{\bar{\tau}}}\left(\int_{Q_{2 r}(x)}|u|^{2} d x\right)^{\tau^{\star}+1}\right\} .
\end{aligned}
$$

Now, we just need to repeat the steps after (4.13) in the proof of Lemma 4.1 and Lemma 4.2 is established.

Lemma 4.3. Let $u \in C^{1}\left(\mathbf{R}^{2}, \mathbf{R}^{2}\right)$ be an entire weak solution of (1.1), then the following results hold:

(a) If $2 \leq \tau^{\prime} \leq 3$ and $u \in L^{p}\left(\mathbf{R}^{2}, \mathbf{R}^{2}\right), 1<p<2$, then $u$ must be the zero vector.

(b) If $3<\tau^{\prime}<4$ and $u \in L^{p}\left(\mathbf{R}^{2}, \mathbf{R}^{2}\right), \tau^{\prime}-2<p<2$, then $u$ must vanish identically.

Proof. For any $q>2$, by Lemma 2.8 we have

$$
\int_{Q_{R}\left(x_{0}\right)}|u|^{q} d x \leq C(q)\left\{R^{2}\left(\int_{Q_{R}\left(x_{0}\right)}|D u|^{2} d x\right)^{\frac{q}{2}}+\frac{R^{2}}{R^{q}}\left(\int_{Q_{R}\left(x_{0}\right)}|u|^{2} d x\right)^{\frac{q}{2}}\right\},
$$

from which, together with Lemma 2.8, it follows that

$$
\int_{Q_{R}\left(x_{0}\right)}|u|^{q} d x \leq C(q)\left\{R^{2}\left(\int_{Q_{R}\left(x_{0}\right)}|\varepsilon(u)|^{2} d x\right)^{\frac{q}{2}}+\frac{R^{2}}{R^{q}}\left(\int_{Q_{R}\left(x_{0}\right)}|u|^{2} d x\right)^{\frac{q}{2}}\right\} .
$$

Combining (4.16) and (4.22) we obtain

$$
\begin{aligned}
\int_{Q_{R}\left(x_{0}\right)}|u|^{q} d x & \leq C(q)\left\{R^{2}\left(\int_{Q_{R}\left(x_{0}\right)} h(\varepsilon(u)) d x\right)^{\frac{q}{2}}+\frac{R^{2}}{R^{q}}\left(\int_{Q_{R}\left(x_{0}\right)}|u|^{2} d x\right)^{\frac{q}{2}}\right\} \\
& \leq C(\tau, q)\left\{\frac{R^{2}}{R^{q}}\left(\int_{Q_{2 R}\left(x_{0}\right)}|u|^{2} d x\right)^{\frac{q}{2}}+\frac{R^{2}}{R^{\bar{\tau} \frac{q}{2}}}\left(\int_{Q_{2 R}\left(x_{0}\right)}|u|^{2} d x\right)^{\frac{q}{2}}\right. \\
& \left.+\frac{R^{2}}{R^{q}}\left(\int_{Q_{2 R}\left(x_{0}\right)}|u|^{2} d x\right)^{q}+\frac{R^{2}}{R^{\bar{\tau} \frac{q}{2}}}\left(\int_{Q_{2 R}\left(x_{0}\right)}|u|^{2} d x\right)^{\frac{q}{2}\left(\tau^{\star}+1\right)}\right\} .
\end{aligned}
$$


On the other hand, for $1<p<2$, Hölder's inequality gives

$$
\int_{Q_{2 R}\left(x_{0}\right)}|u|^{2} d x \leq\left(\int_{Q_{2 R}\left(x_{0}\right)}|u|^{p} d x\right)^{\frac{1}{p^{\prime}}}\left(\int_{Q_{2 R}\left(x_{0}\right)}|u|^{q} d x\right)^{\frac{1}{q^{\prime}}}
$$

where $\frac{1}{p^{\prime}}=\frac{1-\frac{2}{q}}{p\left(\frac{1}{p}-\frac{1}{q}\right)}, \frac{1}{q^{\prime}}=\frac{\frac{2}{p}-1}{q\left(\frac{1}{p}-\frac{1}{q}\right)}$. Putting together the estimates (4.23) and (4.24) we deduce

$$
\begin{aligned}
\int_{Q_{R}\left(x_{0}\right)}|u|^{q} d x \leq & C(\tau, q)\left\{\frac{R^{2}}{R^{q}}\left(\int_{Q_{2 R}\left(x_{0}\right)}|u|^{p} d x\right)^{\frac{q}{2 p^{\prime}}}\left(\int_{Q_{2 R}\left(x_{0}\right)}|u|^{q} d x\right)^{\frac{q}{2 q^{\prime}}}\right. \\
& +\frac{R^{2}}{R^{\tau} \frac{q}{2}}\left(\int_{Q_{2 R}\left(x_{0}\right)}|u|^{p} d x\right)^{\frac{q}{2 p^{\prime}}}\left(\int_{Q_{2 R}\left(x_{0}\right)}|u|^{q} d x\right)^{\frac{q}{2 q^{\prime}}} \\
& +\frac{R^{2}}{R^{q}}\left(\int_{Q_{2 R}\left(x_{0}\right)}|u|^{p} d x\right)^{\frac{q}{p^{\prime}}}\left(\int_{Q_{2 R}\left(x_{0}\right)}|u|^{q} d x\right)^{\frac{q}{q^{\prime}}} \\
& \left.+\frac{R^{2}}{R^{\tau^{2}}}\left(\int_{Q_{2 R}\left(x_{0}\right)}|u|^{p} d x\right)^{\frac{q\left(\tau^{\star}+1\right)}{2 p^{\prime}}}\left(\int_{Q_{2 R}\left(x_{0}\right)}|u|^{q} d x\right)^{\frac{q\left(\tau^{\star}+1\right)}{2 q^{\prime}}}\right\} .
\end{aligned}
$$

Since $\lim _{q \rightarrow \infty} \frac{q}{q^{\prime}}=\lim _{q \rightarrow \infty} \frac{\frac{2}{p}-1}{\frac{1}{p}-\frac{1}{q}}=2-p$ and $\lim _{q \rightarrow \infty} \frac{q\left(\tau^{\star}+1\right)}{2 q^{\prime}}=\frac{2-p}{4-\tau^{\prime}}$, if $2 \leq \tau^{\prime} \leq 3,1<p<2$ or $3<\tau^{\prime}<4, \tau^{\prime}-2<p<2$, then we always have $2-p<1$ and $\frac{2-p}{4-\tau^{\prime}}<1$. Hence we can choose $q$ large enough s.t. $\frac{q}{q^{\prime}}<1, \frac{q\left(\tau^{\star}+1\right)}{2 q^{\prime}}<1$. Thus, for any $\delta>0$, using Young's inequality in (4.25) we have

$$
\begin{aligned}
\int_{Q_{R}\left(x_{0}\right)}|u|^{q} d x \leq \delta \int_{Q_{2 R}\left(x_{0}\right)}|u|^{q} d x+C(\tau, \delta, q)\left\{\left(\frac{R^{2}}{R^{q}}\right)^{\alpha_{1}}\left(\int_{Q_{2 R}\left(x_{0}\right)}|u|^{p} d x\right)^{\beta_{1}}\right. \\
+\left(\frac{R^{2}}{R^{\bar{\tau} \frac{q}{2}}}\right)^{\alpha_{2}}\left(\int_{Q_{2 R}\left(x_{0}\right)}|u|^{p} d x\right)^{\beta_{2}}+\left(\frac{R^{2}}{R^{q}}\right)^{\alpha_{3}}\left(\int_{Q_{2 R}\left(x_{0}\right)}|u|^{p} d x\right)^{\beta_{3}} \\
\left.+\left(\frac{R^{2}}{R^{\bar{\tau} \frac{q}{2}}}\right)^{\alpha_{4}}\left(\int_{Q_{2 R}\left(x_{0}\right)}|u|^{p} d x\right)^{\beta_{4}}\right\}
\end{aligned}
$$

where $\alpha_{i}, \beta_{i}, 1 \leq i \leq 4$, are positive numbers.

By Lemma 2.6 we obtain

$$
\begin{aligned}
\int_{Q_{R}\left(x_{0}\right)}|u|^{q} d x \leq C(\tau, q)\left\{\left(\frac{R^{2}}{R^{q}}\right)^{\alpha_{1}}\left(\int_{Q_{2 R}\left(x_{0}\right)}|u|^{p} d x\right)^{\beta_{1}}\right. \\
+\left(\frac{R^{2}}{R^{\bar{\tau} \frac{q}{2}}}\right)^{\alpha_{2}}\left(\int_{Q_{2 R}\left(x_{0}\right)}|u|^{p} d x\right)^{\beta_{2}}+\left(\frac{R^{2}}{R^{q}}\right)^{\alpha_{3}}\left(\int_{Q_{2 R}\left(x_{0}\right)}|u|^{p} d x\right)^{\beta_{3}} \\
\left.+\left(\frac{R^{2}}{R^{\bar{\tau} \frac{q}{2}}}\right)^{\alpha_{4}}\left(\int_{Q_{2 R}\left(x_{0}\right)}|u|^{p} d x\right)^{\beta_{4}}\right\} .
\end{aligned}
$$

Letting $R \rightarrow \infty$ in (4.27) and observing $\int_{\mathbf{R}^{2}}|u|^{p} d x<\infty$ we deduce that

$$
\int_{\mathbf{R}^{2}}|u|^{q} d x=0
$$

therefore, $u=0$, the proof is complete. 
Lemma 4.4. Let $u \in C^{1}\left(\mathbf{R}^{2}, \mathbf{R}^{2}\right)$ be an entire weak solution of (1.1). Then the following results hold

(a) If $2 \leq \tau^{\prime}<4$ and $u \in L^{p}\left(\mathbf{R}^{2}, \mathbf{R}^{2}\right), p \geq 2$, then $u$ must the zero vector.

(b) If $\tau^{\prime} \geq 4$ and $u \in L^{\tau^{\prime}}\left(\mathbf{R}^{2}, \mathbf{R}^{2}\right)$, then $u$ vanishes identically.

Proof. For obtaining $(a)$, we will first show that the integrals of $h(|\varepsilon(u)|)$ and $|D u|^{2}$ are both locally uniformly bounded. In fact, for any $x_{0} \in \mathbf{R}^{2}$, choosing $R=2$ in (4.16) and recalling the conditions (2.1) and (2.2) we obtain

$$
\int_{Q_{2}\left(x_{0}\right)}|\varepsilon(u)|^{2} d x \leq C \int_{Q_{2}\left(x_{0}\right)} h(|\varepsilon(u)|) d x \leq C\left(\|u\|_{L^{p}}, \tau\right), \forall x_{0} \in \mathbf{R}^{2},
$$

from which, together with Lemma 2.4, it follows

$$
\int_{Q_{2}\left(x_{0}\right)}|D u|^{2} d x \leq C\left(\|u\|_{L^{p}}, \tau\right), \forall x_{0} \in \mathbf{R}^{2} .
$$

Secondly, observing that we just need the fact that the integrals of $h(|\varepsilon(u)|)$ and $|D u|^{2}$ are both locally uniformly bounded in order to carry out the proof of Lemma 3.1 we deduce

$$
\begin{aligned}
\int_{Q_{1}\left(x_{0}\right)} W d x \leq & C\left(\|u\|_{L^{p}}, \tau\right)\left\{\int_{Q_{2}\left(x_{0}\right)} h(|\varepsilon(u)|) d x\right. \\
& \left.+\int_{Q_{2}\left(x_{0}\right)}|D u|^{2} d x+1+\int_{Q_{2}\left(x_{0}\right)}|u| d x\right\} .
\end{aligned}
$$

Putting together the estimates (4.28), (4.29), (4.30) and recalling the condition $u \in$ $L^{p}\left(\mathbf{R}^{2}, \mathbf{R}^{2}\right), p \geq 2$ we have

$$
\int_{Q_{1}\left(x_{0}\right)}\left|D^{2} u\right|^{2} d x \leq C \int_{Q_{1}\left(x_{0}\right)}|D \varepsilon(u)|^{2} d x \leq C \int_{Q_{1}\left(x_{0}\right)} W d x \leq C\left(\|u\|_{L^{p}}, \tau\right),
$$

where the inequality $\left|D^{2} u(x)\right| \leq C|D \varepsilon(u)(x)|$ is used.

In view of (4.31) and Sobolev's imbedding theorem we know $u \in L^{\infty}\left(\mathbf{R}^{2}, \mathbf{R}^{2}\right)$. Hence, by Lemma 2.7, we know that $u$ must be a constant vector. Since $u \in$ $L^{p}\left(\mathbf{R}^{2}, \mathbf{R}^{2}\right)$, then $u=0$.

Thus the proof of $(a)$ is complete. For $(b)$, observing Lemma 4.1 and the condition $u \in L^{\tau^{\prime}}\left(\mathbf{R}^{2}, \mathbf{R}^{2}\right)$, we just need to repeat the above steps.

Remark 4.1. If $u \in C^{1}\left(\mathbf{R}^{2}, \mathbf{R}^{2}\right)$ is an entire weak solution of (1.1) and

$$
\int_{\mathbf{R}^{2}} h(|\varepsilon(u)|) d x<\infty
$$

$u$ may not be a constant vector. One counter example is given by $u_{1}=-y, u_{2}=x$. But if the integrals of $|u|^{p}, p \geq 1$ and $h(|\varepsilon(u)|)$ are both locally uniformly bounded, then by Lemma 3.1 we know that $u \in L^{\infty}\left(\mathbf{R}^{2}, \mathbf{R}^{2}\right)$. Hence $u$ is a constant vector.

Acknowledgement. The author wants to express his gratitude to the referee of the paper for valuable comments and suggestion, based on which, the paper was revised. He also thanks the Academy of Finland for kind support. 


\section{References}

[BFZ05] Bildhauer, M., M. Fuchs, and X. Zhong: A lemma on the higher integrability of functions with applications to the regularity theory of two-dimensional generalized Newtonian fluids. - Manuscripta Math. 116:2, 2005, 135-156.

[BFZ13] Bildhauer, M., M. Fuchs, and G. Zhang: Liouville-type theorems for steady flows of degenerate power law fluids in the plane. - J. Math. Fluid Mech. 15:3, 2013, 583-616.

[Eva98] Evans, L. C.: Partial differential equations. - Grad. Stud. Math. 19, Amer. Math. Soc., Providence, RI, 1998.

[Fuc12a] Fuchs, M.: Stationary flows of shear thickening fluids in 2D. - J. Math. Fluid Mech. 14:1, 2012, 43-54.

[Fuc12b] Fuchs, M.: Liouville theorems for stationary flows of shear thickening fluids in the plane. - J. Math. Fluid Mech. 14:3, 2012, 421-444.

[Fuc14] Fuchs, M.: - Variations on Liouville's theorem in the setting of stationary flows of generalized Newtonian fluids in the plane. - Amer. Math. Soc. Transl. 232:2, 2014, 7998.

[FS00] Fuchs, M., and G. SEREGin: Variational methods for problems from plasticity theory and for generalized Newtonian fluids. - Lecture Notes in Math. 1749, Springer-Verlag, Berlin, 2000.

[FZ12] Fuchs, M., and G. Zhang: Liouville theorems for entire local minimizers of energies defined on the class $L \log L$ and for entire solutions of the stationary Prandtl-Eyring fluid model. - Calc. Var. Partial Differential Equations 44:1-2, 2012, 271-295.

[Gal94a] GALDI, G. P.: An introduction to the mathematical theory of the Navier-Stokes equations. Volume I. - Springer Tracts in Natural Philosophy 38, Springer-Verlag, New York, 1994.

[Gal94b] GALDI, G. P.: An introduction to the mathematical theory of the Navier-Stokes equations. Volume II. - Springer Tracts in Natural Philosophy 39, Springer-Verlag, New York, 1994.

[GM82] Giaquinta, M., and G. Modica: - Nonlinear systems of the type of the stationary Navier-Stokes system. - J. Reine Angew. Math. 330, 1982, 173-214.

[GT83] Gilbarg, D., and N.S. Trudinger: Elliptic partial differential equations of second order. - Grundlehren Math. Wiss. 224, second edition, Springer-Verlag, Berlin, 1983.

[GW78] Gilbarg, D., and H. F. Weinberger: Asymptotic properties of steady plane solutions of the Navier-Stokes equations with bounded Dirichlet integral. - Ann. Scuola Norm. Sup. Pisa Cl. Sci. (4) 5:2, 1978, 381-404.

[JK14] JIN, B. J., and K. KANG: Liouville theorem for the steady-state non-Newtonian NavierStokes equations in two dimensions. - J. Math. Fluid Mech. 16:2, 2014, 275-292.

[KNSS09] Koch, G., Nadirashvili, N., G. A. Seregin, V. Sverak: Liouville theorems for the Navier-Stokes equations and applications. - Acta Math. 203:1, 2009, 83-105.

[Lad69] Ladyzhenskaya, O.A.: The mathematical theory of viscous incompressible flow. Mathematics and its Applications 2, second English edition, revised and enlarged, Gordon and Breach Science Publishers, New York, 1969.

[MNRR96] Malek, J., J. Necas, M. Rokyta, and Ruzicka, M.: Weak and measure-valued solutions to evolutionary PDEs. - Applied Mathematics and Mathematical Computation 13, Chapman \& Hall, London, 1996.

[Tem83] Temam, R.: Mathematical problems in plasticity. - Méthodes Mathématiques de l'Informatique 12, Gauthier-Villars, Montrouge, 1983.

[Zha13] ZhANG, G.: A note on Liouville theorem for stationary flows of shear thickening fluids in the plane. - J. Math. Fluid Mech. 15:4, 2013, 771-782.

Received 7 January 2015 • Revised received 23 March $2015 \bullet$ Accepted 29 March 2015 